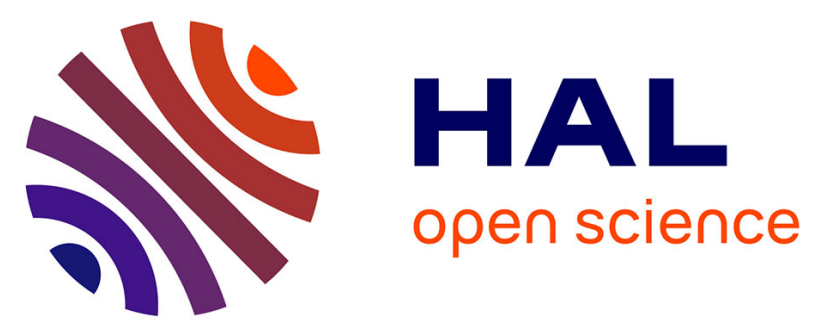

\title{
A variant in MRPS14 (uS14m) causes perinatal hypertrophic cardiomyopathy with neonatal lactic acidosis, growth retardation, dysmorphic features and neurological involvement
}

Christopher Jackson, Martina Huemer, Ramona Bolognini, Franck Martin, Gabor Szinnai, Birgit Donner, Uwe Richter, Brendan Battersby, Jean-Marc Nuoffer, Anu Suomalainen, et al.

\section{- To cite this version:}

Christopher Jackson, Martina Huemer, Ramona Bolognini, Franck Martin, Gabor Szinnai, et al.. A variant in MRPS14 (uS14m) causes perinatal hypertrophic cardiomyopathy with neonatal lactic acidosis, growth retardation, dysmorphic features and neurological involvement. Human Molecular Genetics, 2019, 28 (4), pp.639-649. 10.1093/hmg/ddy374 · hal-02119070

\section{HAL Id: hal-02119070 \\ https://cnrs.hal.science/hal-02119070}

Submitted on 5 Oct 2020

HAL is a multi-disciplinary open access archive for the deposit and dissemination of scientific research documents, whether they are published or not. The documents may come from teaching and research institutions in France or abroad, or from public or private research centers.
L'archive ouverte pluridisciplinaire HAL, est destinée au dépôt et à la diffusion de documents scientifiques de niveau recherche, publiés ou non, émanant des établissements d'enseignement et de recherche français ou étrangers, des laboratoires publics ou privés. 
A variant in MRPS14 (uS14m) causes perinatal hypertrophic cardiomyopathy with neonatal lactic acidosis, growth retardation, dysmorphic features and neurological involvement

Christopher B Jackson ${ }^{1}$, Martina Huemer ${ }^{2,3}$, Ramona Bolognini ${ }^{4}$, Franck Martin ${ }^{5}$, Gabor Szinnai $^{3,6}$, Birgit C Donner ${ }^{7}$, Uwe Richter ${ }^{8}$, Brendan J Battersby ${ }^{8}$, Jean-Marc Nuoffer ${ }^{9,10}$, Anu Suomalainen ${ }^{1,11}$, André Schaller ${ }^{4 *}$

\footnotetext{
${ }^{1}$ Research Programs Unit, Molecular Neurology, Biomedicum Helsinki, University of Helsinki, Finland

${ }^{2}$ Division of Metabolism and Children's Research Center, University Children's Hospital Zürich, Switzerland

${ }^{3}$ University Children's Hospital Basel, University of Basel, Switzerland

4 Division of Human Genetics, Department of Pediatrics, Inselspital, Bern University Hospital, University of Bern, Switzerland

${ }^{5}$ CNRS, Architecture et Réactivité de l’ARN, Université de Strasbourg, UPR 9002, F-67000 Strasbourg, France

${ }^{6}$ Division of Pediatric Endocrinology, University Children's Hospital Basel, Switzerland

${ }^{7}$ Division of Cardiology, University of Basel, Switzerland

${ }^{8}$ Institute of Biotechnology, University of Helsinki, Helsinki, Finland

${ }^{9}$ Institute of Clinical Chemistry, University of Bern, Inselspital, Switzerland

${ }^{10}$ Division of Endocrinology Diabetology and Metabolism, University Children's Hospital, University of Bern

${ }^{11}$ Neuroscience Center, University of Helsinki, Finland.
}

\section{Corresponding author:}

André Schaller

Division of Human Genetics

Department of Pediatrics

University Hospital Bern, Switzerland

Freiburgstrasse 15, 3010 Bern

Tel.: +41316327845

Fax: +41316329484

E-mail: andre.schaller@insel.ch 


\begin{abstract}
Dysfunction of mitochondrial translation is increasingly an important molecular cause of human disease, but structural defects of mitochondrial ribosomal subunits are rare. We used next-generation sequencing to identify a homozygous variant in the mitochondrial small ribosomal protein 14 (MRPS14, uS14m) in a patient manifesting with perinatal hypertrophic cardiomyopathy, growth retardation, muscle hypotonia, elevated lactate, dysmorphy and mental retardation. In skeletal muscle and fibroblasts from the patient there was biochemical deficiency in complex IV of the respiratory chain. In fibroblasts mitochondrial translation was impaired and ectopic expression of a wild type MRPS14 cDNA functionally complemented this defect. Surprisingly, the mutant uS14m was stable and did not affect assembly of the small ribosomal subunit. Instead, structural modeling of the uS14m mutation predicted a disruption to the ribosomal mRNA channel. Collectively, our data demonstrates pathogenic mutations in MRPS14 can manifest as a perinatal-onset mitochondrial hypertrophic cardiomyopathy with a novel molecular pathogenic mechanism that impairs the function of mitochondrial ribosomes during translation elongation or mitochondrial mRNA recruitment rather than assembly.
\end{abstract}




\section{Introduction}

Mitochondria contain their own genome and retain an intrinsic bacteriophage/bacteria-like replication and translation machinery. 13 proteins need to be expressed from the mitochondrial genome (mtDNA) to produce a functional respiratory system and ATP synthase for oxidative phosphorylation. Over the last decade, genetic mutations that disrupt the function of mitochondrial protein synthesis have been identified with human disease. Surprisingly, very few of these mutations have been identified in the 82 structural subunits of the mitochondrial small and large ribosomal subunits (1). Defects in mitochondrial translation lead to an exceptionally wide-range of clinical phenotypes. Most affected individuals show severe early-onset clinical presentations, often with a fatal outcome (2). These gene defects cause typically either a combined deficiency of respiratory chain or an isolated complex IV defect, manifesting in multiple organs or in a tissue-specific manner (3). Currently, the molecular basis by which oxidative phosphorylation defects can manifest with such exceptional clinical heterogeneity cannot be explained.

The human mitochondrial ribosome is a $55 \mathrm{~S}$ particle, composed of two subunits, a small $28 \mathrm{~S}$ and a large 39S subunit. The small mitochondrial ribosomal subunit (SSU) is composed of a $12 \mathrm{~S}$ ribosomal RNA molecule (rRNA) and 30 nuclear-encoded mitochondrial ribosomal proteins (MRPs), whereas the large subunit (LSU) includes 16S rRNA and 52 nuclearencoded MRPs and either tRNA ${ }^{\text {Phe }}$ or tRNA ${ }^{\text {Val }}$ (4-7). Both rRNAs, essential for ribosomal integrity and function, are encoded by the mitochondrial genome. Many of the protein subunits of the mitochondrial ribosome (mitoribosome) share structural homology with bacterial counterparts, but 35 out of the 80 MRPs do not and are specific to mammalian mitoribosomes acquired during mitochondrial evolution of mitochondrial gene expression $(8$, 9). Despite recent advances in understanding mitoribosomal structure, the functions of the individual subunits in protein synthesis are still poorly known $(4,10)$. 
Here we report that a variant in MRPS14, encoding the mitochondrial ribosomal protein S14, is a novel cause for progressive infantile mental retardation, cachexia, muscular hypotonia, hypertrophic cardiomyopathy, elevated lactate and a complex IV deficiency. Furthermore, we present biochemical and protein modeling data providing information of MRPS14 roles on mitoribosome.

\section{Results}

\section{Patient history}

The female proband was the first living child from distantly related Turkish parents. The mother suffered from recurrent depressive episodes. The father had experienced a single episode of rhabdomyolysis followed by undulating plasma creatine phosphokinase (CK) values despite complete clinical restitution. The mother had two spontaneous abortions between gestational weeks 10 and 12. A third pregnancy had been terminated at gestational week 24 due to severe hypertrophic non-compaction cardiomyopathy and intrauterine growth retardation of the female fetus. The index patient was delivered through caesarean section after premature rupture of the membranes at 35 weeks of gestation. The low birth weight $\left(1460 \mathrm{~g} ;<3^{\text {rd }}\right.$ percentile) was attributed to placental insufficiency. APGAR score was $3 / 6 / 8$ at 1/5/10 minutes and the child was admitted to the neonatal intensive care unit due to respiratory insufficiency. Hypertrophic cardiomyopathy, suspected already in the prenatal ultrasound examination, with moderate hypertrophy of the left ventricle and mildly impaired contractility was diagnosed.

Metabolic workup revealed moderately elevated serum lactate $(5-7 \mathrm{mmol} / \mathrm{L}$, normal range $<$ 2mmol/L) and alanine. Organic acids, acylcarnitines, free carnitine, liver transaminases, ammonia, creatinine, complete blood cell counts, and creatinine phosphokinase were repeatedly normal. At 4 months, the child was admitted to hospital following an acute lifethreatening event at home, which was attributed to severe gastroesophageal reflux. Lactate 
was again slightly elevated. Cardiologic workup revealed Wolff-Parkinson-White (WPW syndrome) with an average heart rate of $140 / \mathrm{min}$. Clinical assessment proved generalised muscular hypotonia, failure to thrive and retardation of cognitive and motor development; the EEG was normal.

Respiratory chain enzyme activity analysis of the patient's skeletal muscle sample revealed mitochondrial complex IV deficiency, reduced to $44 \%$ of the controls' mean, whereas the other RC complexes were not affected (Fig.S1). Supportive treatment with carnitine, riboflavin, thiamine, vitamin $\mathrm{C}$ and coenzyme $\mathrm{Q}$ was applied for some months without perceivable positive response. Despite hypercaloric diet, the patient showed failure to thrive. During viral infections or episodes of fever, the child repeatedly experienced acute deterioration, with elevated lactate, metabolic acidosis, and poor condition. However, from the age of two years onwards, the patient's clinical condition stabilized and she started to catch up in growth. Her psychomotor development improved and she learned to walk and talk at the age of 3.5 years. The hypertrophic cardiomyopathy remained stable and caused no clinical problems; episodes of supraventricular tachycardia were not observed.

Presently, at the age of 5 years, the patient walks broad-based, unassisted. She has some dysmorphic features, including retroverted low set ears (Fig.1A). She still has muscular hypotonia, however less prominent. Her language skills are continuously improving, although not reaching the standard of the age. With five feedings of wholesome food, supplemented with hypercaloric drinks, her weight gain and growth are now parallel to the $3^{\text {rd }}$ percentile. Her ECG reveals a preexcitation pattern without supraventricular tachycardia, and 3D speckle tracking shows mild segmental dyskinesia in the basal inferior lateral and lateral regions, consistent with an accessory bundle in the lateral wall of the left ventricle. Echocardiography shows no structural abnormalities, and the cardiomyopathy has completely receded. Other 
functional parameters, including 3D volumetry and strain analysis, are in normal limits. The tissue and pulse wave Doppler measurements show normal diastolic function.

Next-generation MitoExome sequencing revealed a recessive homozygous MRPS14 mutation in the patient

We utilized next-generation MitoExome sequencing for molecular genetic investigations (11). The targeted MitoExome approach analyzed sequences of 1476 nuclear genes encoding proteins with mitochondria-associated functions. Data analysis led to identification of a homozygous variant in MRPS14 (NM_022100), c.322C $>$ T (CADD score 8.03) in exon 3 (Fig.1BC). MRPS14 was the only candidate gene fitting a recessive homozygous trait considering the parental consanguinity. This variant was not reported in any databse (ExaC, gnomAD (12)). The functional impact of the c.322C $>\mathrm{T}$ transition has 8 pathogentic computational verdicts (DANN, GERP, LRT, MetaLR, MetaSVM, MutationAssessor, MutationTaster and PROVEAN (listed in (13)). Confirmation of the variant was performed by Sanger sequencing. Carrier analysis showed that both the parents were heterozygous carriers for the c.322C $>$ T variant (Fig.1C). Human MRPS14 encodes a 128 amino acid long protein that is a structural component of the mitoribosome $\mathrm{SSU}$. The c. $322 \mathrm{C}>\mathrm{T}$ variant is predicted to alter a highly conserved arginine to a cysteine at position 108 in uS14m (Fig.1D). Collectively, our data is consistent with the c.322C $>\mathrm{T}$ MRPS14 variant as the underlying pathogenic cause of the patient's clinical presentation.

\section{Decreased mitochondrial respiration and multiple respiratory chain complex deficiency in patient fibroblasts}

Next, we analysed patient fibroblasts to test whether the c.322C $>\mathrm{T}$ variant was pathogenic and disrupted mitochondrial protein synthesis. Blue-native gel electrophoresis analysis of the 
native respiratory complexes revealed combined deficiency of assembled oxidative phosphorylation complexes, with CI and CIV barely detectable, and CIII and CV markedly decreased (Fig.2A). Steady-state mitochondrial respiratory proteins showed decreased levels of respiratory complex subunits with most substantial decrease in subunits probed for complex I and IV (Fig.2B). The exclusively nuclear-encoded complex II showed no significant reduction compared to controls (Fig.2B). These changes of multiple respiratory chain deficiency were also reflected in base level oxygen consumption, with a markedly deficient ADP-dependent and slightly decreased complex IV-dependent respiration activity (Fig.S2A) and partially distorted mitochondrial structure (Fig.S2B), but no changes in mitochondrial mass in patient cells compared to controls (Fig.S2C). Confirming mitochondrial disease etiology, we probed for steady-state levels of mitochondrial small (SSU) and large (LSU) ribosomal subunits to assess ribosomal integrity. Intriguingly, when we assessed the steady-state levels of mitochondrial small (SSU) and large (LSU) ribosomal subunits we found MRPS14 levels were increased in the patient, whereas all SSU (MRPS10, MRPS18B, MRPS27) and LSU (MRPL11, MRPL37, MRPL44) subunits tested remained unchanged compared to controls (Fig.2C), indicating the the c.322C $>\mathrm{T}$ variant in MRPS14 does not impair the assembly of the SSU.

\section{The c.322C $>T$ variant in MRPS14 does not affect ribosome assembly or 12S rRNA stability, but is essential for mitochondrial translation}

To assess the assembly status of the entire mitoribosome and to confirm insertion of MRPS14 into the SSU, sucrose gradients analysis was performed. Probing against MRPS18B revealed specific and comparable separation of mitoribosomal components in control and patient fibroblasts (Fig.3A). MRPS14 was found both in the SSU and monosome fractions comparable to controls, suggestive of insertion of both normal and mutant protein (Fig.3A). Probing of LSU subunit MRPS27 and LSU subunits MRPL23 and MRPL44 were unchanged 
compared to patients, demonstrating the mitoribosome to be intact in the patient cells.

Northern analysis of the steady-state mtDNA transcript levels showed no reduction in the $12 \mathrm{~S}$ rRNA levels compared to controls, which would indicate instability or assembly deficiency of the SSU (Fig.3B, quantification Fig.S3A). LSU associated 16S rRNA and ATP8/6 mRNA were unchanged relative to total RNA (Fig.3B, Fig.S3A). These findings were corroborated by quantitative PCR (Fig.3C). The comparable amount of $12 \mathrm{~S}$ rRNA supported the conclusion that the mutated MRPS14 did not impair assembly or stability of the SSU. However, the role of MRPS14 in the assembly or stability of the small mitoribosomal subunit and effect on on translation of specific mRNAs is unknown. Up to date, mutations reported in mitochondrial ribosomal subunits cause protein instability with subsequent instability or defective assembly of the mitoribosome and its associated rRNAs (14-20). Western blot analysis indicated that the mutant MRPS14 protein was stable, suggesting it to be inserted into the mitoribosome and directly interfere with the mitochondrial translation process rather than affect mitoribosome assembly or stability (Fig.2C).

To directly test whether the $\mathrm{c} .322 \mathrm{C}>\mathrm{T}$ variant in MRPS14 indeed impaired the mitochondrial translation; we labeled the mitochondrial translation products in the fibroblasts by metabolic ${ }^{35}$ S-labelling. Pulse-labelling revealed a general mitochondrial translation defect in the patient's fibroblasts compared to the controls as total $\left[{ }^{35} \mathrm{~S}\right]-\mathrm{met} / \mathrm{cys}$ incorporation was significantly reduced (Fig.3DE). Due to apparent stability of the mitoribosome, we performed a chase experiment to exclude a specific translational interaction or modification to any mitochondrially translated protein resulting in increased instability, which did, however, not show any complete turnover of a specific product (Fig.S3B). Silencing of MRPS14 did not result in a lower steady-state level of MT-CO1, suggesting that the residual amount of wild type mitoribosomes was enough to maintain its levels (Fig.S4). In conclusion, these results indicate that the MRPS14 variant severely inhibits translation efficiency in the patient's fibroblasts without affecting general SSU assembly, the - reported late stage (21) - addition of 
MRPS14 to the SSU itself or 55S monosome assembly.

\section{Modelling the putative role of MRPS14 on 55S ribosome}

Recent high resolution cryo-EM structure of the human mitoribosome initiation complex (22) allowed us to investigate the putative structural impact of the R108C mutation. This structure is especially interesting because it allowed a careful assessment of the position of this residue and its integration translation initiation complex containing a tRNA and mRNA $(4,23)$. $\mathrm{uS} 14 \mathrm{~m}$ is part of the head of the SSU forming a cluster with MRPS24 and uS10m similar to the bacterial counterpart (with the exception of bS6m and uS12m). Mitochondrial specific Nterminal amino extension (NTE) and C-terminal amino acid extension (CTE) are unique features of 15 of the 31 SSU subunits (with the exception of bS6m and uS12m), which are homologous to their bacterial counterpart. They are of varying length and potentially form solvent interaction sites. The NTE of uS14m is located to the solvent side, as is the NTE and CTE of uS10m. Interestingly, uS14m is located in the mRNA entrance channel on the small ribosomal subunit at the interface with the large subunit (Fig.4A). In this structure, the distance between R108 and the tRNA anticodon is above $30 \AA$ (Fig. 4B). The lateral chain of the amino acid R108 affected in the patient interacts with both the small subunit ribosomal protein $\mathrm{uS} 10 \mathrm{~m}$ on one side and the sugar phosphate backbone of residue A579 of 12S rRNA on the other side (Fig.4C). Therefore, R108 occupies a key position that links the 12S rRNA to ribosomal protein $\mathrm{uS} 10 \mathrm{~m}$. A cysteine at position 108 would be predicted to disrupt both interactions, thereby probably leading to an altered conformation of the mRNA channel or the ribosome decoding center (Fig. 4D). Such a model is consistent with our findings that only mitochondrial translation elongation or mitochondrial mRNA recruitment is disrupted in patient fibroblasts and not the assembly of mitoribosomes. 


\section{Wild-type MRPS14 rescues the respiratory chain deficiency and mitochondrial translation defect in patient cells}

To directly test the pathogenicity of the c.322C $>\mathrm{T}$ variant in MRPS14, we cloned the wild type and variant MRPS14 cDNAs to a lentiviral expression system. Both MRPS14 cDNAs were transduced into the patient and control fibroblasts. Overexpression of the wild type MRPS14 cDNA in the patient fibroblasts complemented the steady-state protein levels of MT$\mathrm{CO} 1$ and MT-CO2 and mitochondrial translation (Fig.5A). In contrast, overexpressing the mutation in the patient background failed to rescue the defect in mitochondrial protein synthesis (Fig. 5A) Surprisingly, the overexpression of the R108C mutant in control fibroblasts generated a dominant-negative phenotype, reducing MT-CO1 and MT-CO2 abundance (Fig.5A). To corroborrate these findings, metabolic ${ }^{35} \mathrm{~S}$-pulse-labelling in the same transduced fibroblasts lines was performed (Fig.5B). As suggested by rescued steady-state MT-CO1 and MT-CO2 levels, mitochondrial translation capacity was complemented in patient cells overexpressing wild type with MRPS14 cDNA (Fig.5B).

This would suggest the mutant protein was assembled into the ribosome and is critical for mitochondrial protein synthesis. Together, these data support the pathogenicity of the c. 322C $>\mathrm{T}$ MRPS14 mutation as the underlying cause of the biochemical defect in oxidative phosphorylation in the patient.

\section{Discussion}

Here, we report a novel pathogenic mutation in MRPS14 that manifests as a perinatal mitochondrial hypertrophic cardiomyopathy. The pathogenic role was supported by 1) segregation in the family; 2) high conservation of the mutation site and modeling-based prediction of severe effects of the mutation in intraribosomal interactions; 3) patient's clinical and cellular phenotype mimicking those of other mitochondrial ribosome defects; 4) functional complementation of the biochemical defects with overexpression of the wild type 
protein. This is the first reported pathogenic mitoribosomal defect not affecting the assembly or stability of the mitoribosome, evidencing that the mitoribosome allows incorporation of mutant proteins and instead, disrupt the function of the mitoribosome during translation elongation or mitochondrial mRNA recruitment.

Approximately 80 ribosomal proteins are assembled into the mitochondrial 55S ribosome and surprisingly few have been associated with human diseases or are considered possibly disease-causing (Table 1.). This suggests that many potential mutations are so severe, that they result in early embryonic lethality. Consistent with this hypothesis, the presented family had a history of repeated miscarriages. Previously, human disorders have been associated with MRPS2 (24), MRPS7 [11], MRPS16 [12], MRPS22 [13, 14], MRPS23 [15] and MRPS34 (14) of the small mitochondrial ribosomal subunit and mutations in MRPL3 [16], MRPL12 [17] and MRPL44 [18] encoding proteins of the large ribosomal subunit. These defects often are associated with neonatal hypertrophic cardiomyopathies, similar to our patient. However, the fetal manifestation in the current patient and her sibling is among the earliest manifestations reported, mimicking that of an alanine tRNA synthetase defect (25).

Clinically, patients with defects of the mitochondrial ribosome often present with hypertrophic cardiomyopathy, which in our patient was accompanied by mental retardation, muscle hypotonia, dysmorphism and neurological features with lactic acidosis. Defects to mitochondrial protein synthesis universally disrupt the synthesis of the 13 mtDNA-encoded subunits required for oxidative phosphorylation, and yet, are associated with a broad heterogenous clinical presentation (Table 1). All of the reported cases of mitoribosomal subunit defects manifest early in childhood and are often fatal. However, some can survive beyond the critical infantile years with an apparently stable cardiac phenotype (20). The same natural history is observed in our patient. The molecular mechanisms by which cardiac tissue might compensate for a severe mitochondrial translation defect remains to be discovered. Reported defects of the mitochondrial SSU lead to protein instability and cause impaired 
assembly of the small $28 \mathrm{~S}$ ribosomal subunit and reduction of $12 \mathrm{~S}$ rRNA, which is degraded if not incorporated to SSU during assembly $(15,19,26-28)$. We found mutant MRPS14 to be stable, strongly supporting insertion of the mutant protein into the mitoribosome, without affecting gross ribosomal assembly or its stability. It is possible, however, that the mutated patient mitoribosome lacks subunits not essential for assembly not resolved on sucrose gradients. Whether complete ablation of MRPS14 would cause SSU assembly or stability defects is still unknown. Suprisingly, overexpression of mutant MRPS14 protein phenocopied the patients biochemical findings. Whether the increased steady-state level of MRPS14 in patient cells is a compensatory effect, however, could not be conclusively addressed nor could the ratio of mutant and wild type MRPS14 be determined. We therefore assigned this dominant-additive effect of mutant MRPS14 to its overexpression. Although the heterozygous parents were subclinical, the father presented with a single episode of rhadomyolysis and varying abnormal CK values, suggesting a possible partial dominant negative effect.

Our protein modeling predicted MRPS14 to be located in the mRNA channel, a core functional centre of the ribosome, with close contact with both the mRNA transcript and the $12 \mathrm{~S}$ rRNA. This proposes a possible role for MRPS14 to contribute to the recruitment and guidance of the leaderless mitochondrial mRNAs to the ribosome and resulting impaired translation efficiency. This function would correspond well with the functional defects detected in the patient's fibroblasts, which were able to produce translation products, but with severely reduced efficiency.

Dysmorphic features are common to mitochondrial disease and are described in other patients with SSU gene mutation, but have led to the hypothesis that they have an additional role in development. Furthermore, MRPs have been reported to contribute to apoptosis and regulation of cell proliferation, and even associated with neoplasms (29-33). We cannot exclude contribution of such additional functions to the patient's phenotype. However, our data implicate that MRPS14 is crucial for a highly functional mitochondrial translation 
machinery and thereby oxidative phosphorylation, two essential functions sufficient to explain the leading clinical manifestations of the patient, including the cardiomyopathy.

We report MRPS14 as a novel gene causing mitochondrial hypertrophic cardiomyopathy with dysmorphic features and neurologic involvement, with potential cause of a lethal phenotype in affected fetuses.

\section{Materials and Methods}

\section{OXPHOS assay}

Activity measurements of OXPHOS complexes were performed in mitochondria of skeletal muscle homogenates (supernatants pelleted by centrifugation at $600 \mathrm{xg}$ ) as previously (34). Individual respiratory chain complex activities and the activity of the citrate synthase (CS), used as marker enzyme were measured spectrophotometrically using a UV-1601 described spectrophotometer (Shimadzu) in $1 \mathrm{ml}$ sample cuvettes maintained at $30^{\circ} \mathrm{C}$ according to Birch-Machin [20]. Values were determined by measuring the differences of the complex activity levels in the presence and the absence of specific inhibitors, expressed as ratio to the marker enzyme CS (mU/mU citrate synthase) as described elsewhere [22]. Muscle biopsies of patients without a biochemical and clinical suspicion of a mitochondrial disorder served as a control.

\section{DNA extraction}

DNA was extracted from EDTA-blood from the patient and its parents using a Prepito DNA Blood600 Kit according to the manufacturer's instructions (PerkinElmer). DNA extracted from EDTA-blood was used as template to produce northern blot probes.

\section{Mitochondrial transcript quantification}

RNA from cultured cells was extracted using the Qiagen miRNA kit including a DNase digestion step. Two $\mu \mathrm{g}$ of total RNA was used to generate cDNA using Maxima first-strand 
cDNA synthesis kit (Thermo Scientific). Quantitative real-time PCR amplification of cDNA was performed with IQ SybrGreen kit (Bio-Rad) on CFX96 Touch qPCR system (Bio-Rad). Relative expressions were normalised to $\beta$-actin or 18S rRNA. (Primer sequences Table S1).

\section{Library preparation, targeted sequencing and data analysis}

Next generation sequencing was performed via a MitoExome panel (11). Briefly, $1 \mu \mathrm{g}$ genomic DNA was sheared into fragments of about 300bp using Covaris M220 Focused Ultrasonicator (Covaris) according to the manufacturer`s protocol. Library preparation was performed with the Kapa Library Preparation Kit (Kapa Biosystems) according to manufacturer`s instructions. In solution capturing (NimbleGen, Roche) was used to capture the target regions of 1476 genes (MitoExome). 150 bp paired-end sequencing was performed on a HiSeq2000 (Illumina). Sequence data analysis was perfomred using CLC Genomics Workbench v7.0.3 (CLCbio, Qiagen) for read alignment to the human reference genome GRCh38/hg19, local realignment and variant calling. Variants, which were intronic, synonymous, with a coverage $<20$ and with a minor allele frequency (MAF) of $>1 \%$ were excluded and non-synonymous variants predicted as deleterious (PolyPhen), damaging (SIFT) or with unknown consequences with a MAF of $\leq 1 \%$ were retained.

\section{Molecular genetics}

Analysis of the prioritized homozygous variant in MRPS14 was performed by Sanger sequencing. Exon 3 of MRPS14 (NM_022100) was amplified using genomic DNA as template and was sequenced on an ABI Prism 3500XL Genetic Analyzer (Applied Biosystems) using BigDye Terminator v3.1 chemistry (Applied Biosystems). Sequence data were analyzed with SeqAnalysis 6 (Applied Biosystems) and Sequence pilot v4.3 (JSI medical systems). 


\section{Cell culture}

Primary fibroblast cultures derived from a skin biopsy of the patient and control subjects were cultured in DMEM (1X) + GlutaMAX-I containing $1 \mathrm{~g} / \mathrm{L}$ D-glucose and $110 \mathrm{mg} / \mathrm{L}$ sodium pyruvate (Gibco, Life technologies), supplemented with 10\% fetal bovine serum (FBS) (Gibco, Life technologies), non-essential amino acids (NEAA) (Gibco, Life technologies), 50 $\mathrm{U} / \mathrm{ml}$ penicillin and $50 \mu \mathrm{g} / \mathrm{ml}$ streptomycin (Gibco, Life technologies), $10 \mu \mathrm{g} / \mathrm{ml}$ chlortetracycline (Sigma) and $2 \mu \mathrm{mol} / \mathrm{L}$ uridine at $37^{\circ} \mathrm{C}$ and $5 \% \mathrm{CO}_{2}$.

\section{RNA isolation and northern blotting}

RNA was isolated from primary fibroblasts by using a QIAgen RNeasy Kit (Qiagen) according to the manufacturer's protocol, including an RNase-free DNase digestion step to remove contaminating DNA. Nothern blot analysis has been done as described before (33). Briefly, RNA (10 $\mu \mathrm{g})$ was separated by a $1.5 \%$ denaturing agarose/formaldehyde gel and transferred by capillary transfer in $20 \mathrm{X}$ SSC to a $0.45 \mu \mathrm{m}$ Hybond-N+ nitrocellulose membrane (Amersham). RNA was covalently bound to the membrane in a UV-crosslinker (UV Stratalinker 1800, Stratagene) and hybridised. Probes for Northern blotting were endlabbeled with gamma ATP using PNK accoding to manufacturers recommendations. Membranes were stripped in $0.5 \%$ SDS at $60^{\circ} \mathrm{C}$ and stored at $4{ }^{\circ} \mathrm{C}$ until pre-hybridization.

\section{Cloning and Lentiviral based complementation assay}

Single-stranded cDNA was used as template to amplify wildtype and mutant MRPS14 including a NheI overhang for cloning. Amplified RT-PCR products were cloned into the lentiviral vector pLVEF1a-MCS-IRES-Puro (Biosettia) to obtain pLV-EF1a-wtMRPS14IRES-Puro and pLV-EF1amutMRPS14-IRES-Puro using the NheI site of the MCS and verified by sequencing. Stable lines were produced by selecting transduced fibroblasts with puromycin $(1 \mathrm{ug} / \mathrm{ml})$. Transduction was performed following general protocols. 


\section{SDS and blue native and protein assay}

Total protein was extracted with RIPA buffer using protease, phosphatase inhibitors tablets (ThermoFisher Scientific) and sodium orthovanadate. Protein concentrations were determined using Bradford assay. Proteins were separated by SDS-PAGE using 4-20 \% gradient gels and transferred to PVDF membranes. Blots were blocked with $5 \%[\mathrm{w} / \mathrm{v}]$ milk in Tris buffered saline with $1 \%[\mathrm{v} / \mathrm{v}]$ Tween 20 (TBST) buffer for 1 hour and antibodies were incubated at $4^{\circ} \mathrm{C}$ overnight at 1:1000 dilution in $1 \%[\mathrm{w} / \mathrm{v}]$ milk in TBST buffer. Antibodies used for Western analyses were: MT-CO1 (Abcam, ab14705), MT-CO2 (Abcam, ab110258), SDHA (Abcam, ab14715), ATP5A (Abcam, ab14748), NDUFA9 (Abcam, ab14713), UQCRFS1 (Abcam, ab14746), MRPS14 (Sigma-Aldrich, HPA051087), MRPS27 (Proteintech, 17280-1AP), MRPS10 (Sigma-Aldrich, HPA029134), MRPS18B (Proteintech, 16139-1-AP), MRPL11 (Proteintech, 15543-1-AP), MRPL23 (Proteintech, 11706-1-AP), MRPL44 (Proteintech, 16394-1-AP), MRPL37 (Proteintech, 15190-I-AP), TOM20 (SantaCruz, sc11415), VDAC (Abcam, ab15895), Vinculin (Abcam, ab129002).

Native mitochondrial complexes were prepared from digitonin-solubilised laurylmaltosidetreated fibroblasts as described before (35). Briefly, harvested cells were solubilised in 5 $\mathrm{mg} / \mathrm{ml}$ digitonin for a 0.8 digitonin to total protein ratio in $1 \mathrm{xPBS}$ in the presence of protease inhibitors (ThermoScientific). Solubilised cells were then pelleted, resuspended in $1.5 \mathrm{M}$ aminocaproic acid, $75 \mathrm{mM}$ Bis-tris pH7.0, $10 \mathrm{mM}$ EDTA and treated with $1 \%$ [w/v] laurylmaltoside to liberate respiratory complexes. 10-30 ug of mitochondria-enriched protein was separated on $4-16 \%$ Bis-Tris gels (Life Technologies) for $5 \mathrm{~h}$ and transferred onto PVDF membranes. Blocked membranes were sequentially incubated overnight with MT-CO1 (Abcam, ab14705), SDHA (Abcam, ab14715), ATP5A (Abcam, ab14748), NDUFA9 (Abcam, ab14713), UQCRC2 (Abcam, ab14745) and detected with appropriate secondaryHRP antibodies using chemiluminescence. 


\section{Pulse-labelling of mitochondrial translation products}

Pulse-labelling was performed as described (33) Briefly, fibroblasts were grown in $35 \mathrm{~mm}$ dishes to $60-70 \%$ confluency. Growth media was removed and replaced by methionine/cysteine free medium (Gibco, Life technologies) supplemented with Sodium Pyruvate (Gibco, Life technologies) and pre-incubated before labelling at $37^{\circ} \mathrm{C}$ for $30 \mathrm{~min}$. Cells were incubated for 6 min in met/cys free medium supplemented with 10\% dialyzed FBS (Gibco, Life technologies) and $1 \mathrm{mM}$ Sodium Pyruvate in the presence of $0.5 \mu \mathrm{g} / \mathrm{ml}$ emetine (Sigma, E2375) or $100 \mu \mathrm{g} / \mathrm{ml}$ anisomycin (Sigma A9789) to inhibit cytoplasmic translation prior to the addition of the radiolabel mix. For pulse-labelling $200 \mu \mathrm{Ci} / \mathrm{ml}\left[{ }^{35} \mathrm{~S}\right]-\mathrm{met} / \mathrm{cys}$ (Perkin Elmer) was added to the medium and incubated for $30 \mathrm{~min}$. Cells were washed in growth media and 1xPBS before being trypsinized, collected by centrifugation and resuspended in 1xPBS containing $1 \mathrm{mM}$ PMSF (Sigma) and EDTA-free protease inhibitors (Roche). For chase labelling cells were washed after 30 minutes and incubated in normal growth medium for 3 hours after which cells were washed and harvested. Bradford assay was performed to determine protein concentration according to the manufacturer's protocol (BioRad). $30 \mu \mathrm{g}$ of total protein extracts were separated on $12 \%$ polyacrylamide gels at 20 $\mathrm{mA}$ for $2 \mathrm{~h}$. Gels were stained in Coomassie staining solution ( $1 \mathrm{~g}$ Coomassie brilliant blue in $40 \%[\mathrm{v} / \mathrm{v}]$ methanol, $10 \%[\mathrm{v} / \mathrm{v}]$ glacial acetic acid, $3 \%$ [v/v] glycerol and 47\% [v/v] $\mathrm{H}_{2} \mathrm{O}$ ) overnight and destained in destaining solution $(40 \%[\mathrm{v} / \mathrm{v}]$ methanol, $10 \%[\mathrm{v} / \mathrm{v}]$ glacial acetic acid, $3 \%[\mathrm{v} / \mathrm{v}]$ glycerol and $47 \%[\mathrm{v} / \mathrm{v}] \mathrm{H}_{2} \mathrm{O}$ ) for $2 \mathrm{~h}$. Gels were dried using a vacuum gel dryer Model 583 (BioRad) for $2 \mathrm{~h}$ at $60^{\circ} \mathrm{C}$ and subsequently exposed to a phosphor screen for at least $24 \mathrm{~h}$. Phosphor imaging screens were imaged using a FLA-7000 Typhoon scanner (GE Healthcare) and total protein was imaged using a Chemidoc imaging system (Biorad).

\section{Sucrose gradients}


Sucrose gradients have been prepared as described elsewhere (33). Briefly, 1mg of protein was loaded onto a $16 \mathrm{ml} 10-30 \%$ discontinuous sucrose gradient made up of sucrose and separated at $22000 \mathrm{xg}$ for $16 \mathrm{~h}$. 24 fractions were collected and protein TCA precipitated. Subsequent samples were analysed via SDS-PAGE.

\section{Immunohistochemistry, fluorescence and electron microscopy}

Mitochondrial staining was performed by incubation with Mitotracker CMXRos for 15 minutes in full medium. The medium was replaced and cells replaced in the incubator for 10 minutes in full medium. Cells were rinsed twice with 1xPBS and fixed in ice-cold acetone for 3 minutes and mounted in VectaShield. Immunofluorescence microscopy was performed as described before(36). Samples were imaged in a Zeiss Observer 2.1 with ApoTome. Image contrast was adjusted within the software of the microscope. Electron microscopy was performed as described before (37). Briefly, cells were grown on $13 \mathrm{~mm}$ coverslips, fixed with $2 \%$ GA in phosphate buffer, embedded, sectioned and imaged.

\section{Statistical analysis}

All statistical analyses and graphs were done using PRISM 7.0ab (GraphPad Software, Inc., USA). Quantifications were performed using either FIJI (ImageJ v.2.0.0) or the western blot chemiluminescence signal intensities using ImageLab 5.2.1 (Biorad). Student`s t-test was used to calculate statistical significances were P-values $<0.05$ were considered signficant (* $\mathrm{p} \leq 0.05, * * \mathrm{p} \leq 0.01 . * * * \mathrm{p} \leq 0.001)$.

\section{Acknowledgments}

We are indebted to the family participating in this study. We thank Tuula Manninen for excellent technical assistance and the Electron microscopy unit of the Institute of 
Biotechnology and the Functional Genomics (FuGu) center at the University of Helsinki for their services. This work was supported by the Swiss National Science Foundation [to CJ], the Novartis Foundation for medical biological research [to CJ, AS]; the Academy of Finland and Sigrid Jusélius Foundation [to ASu].

\section{Conflict of Interest Statemant}

No conflicts of interest to declare. 
References

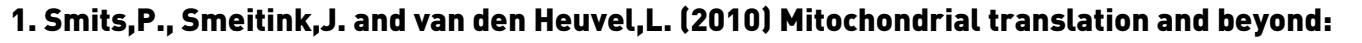

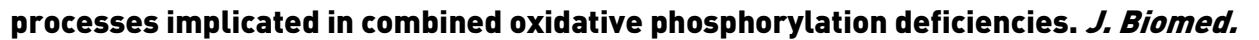
Biotechnol.,[2010,1-24.0

2.[Kemp,J.P.,W5mith,P.M.,IPyle,A.,Weeve,V.C.M.,Tuppen,H.A.L.,Wchara,U.,Talim,B.,Topaloglu,H.,D Holinski-Feder,E.,[Abicht,A.,[et[Bl.L2011)[Nuclear[factors[nvolved[n[mitochondrial[

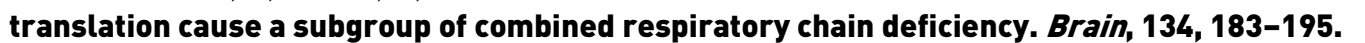

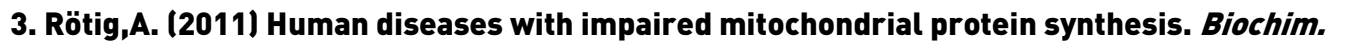
Biophys.[Acta, [807, [198-1205.]

4.AAmunts,A.,Brown,A.,Toots,J.,[Scheres,S.H.W.EndRamakrishnan,V.(12015)[Ribosome.TheQ structure bfitheThuman[mitochondrial[ribosome.DScience,[B48,D5-98.]

5.[Greber,B.J.,Boehringer,D.,Leibundgut,M.,Bieri,P.,Leitner,A.,[Schmitz,N.,_Aebersold,R.End

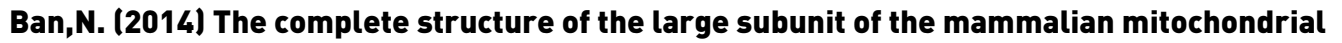
ribosome.DNature, [1515,[283-286.]

6.De[Silva,D.,ITu,Y.-T.,[Amunts,A.,[Fontanesi,F.[TndBarrientos,A.42015][Mitochondrial[ribosome[

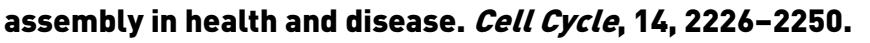

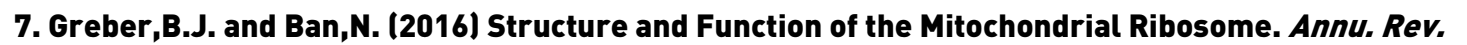
Biochem.,B5, $103-132.0$

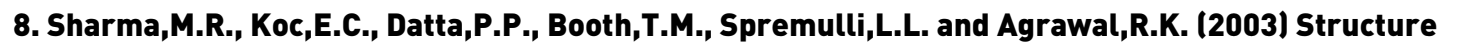

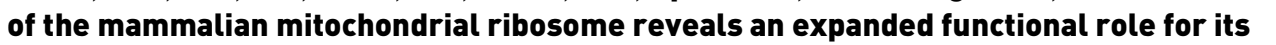

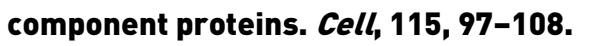

9.Dtt,M.,[Amunts,A.[andBrown,A.12016)Drganization[andRegulation[bfMitochondrial[Protein Synthesis.[Annu.LRev.BBiochem.,B5, $177-101.0$

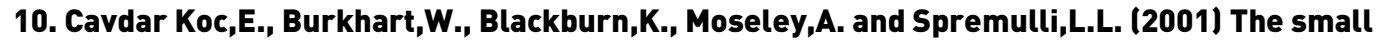

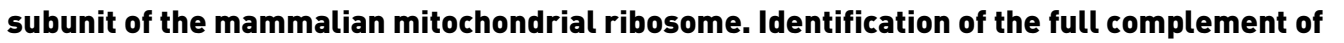

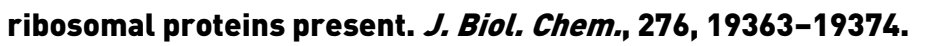

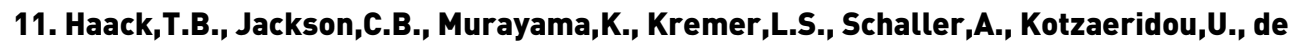

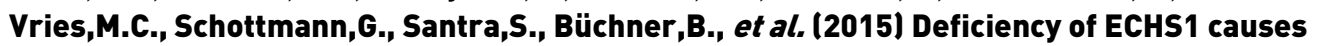
mitochondrial[encephalopathy[with[ardiac[nvolvement.DAnnLlin[Transl[Neurol, $\square$ 10.1002/acn3.189.

12.Lek,M.,[Karczewski,K.J.,IMinikel,E.V.,Wamocha,K.E.,Banks,E.,IFennell,T.,D'Donnell-

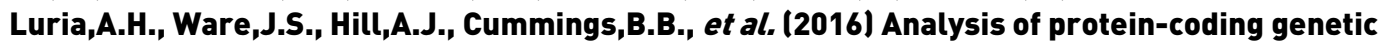
variation[n66,706[humans.[Nature,[536,[285-291.]

13.Doannidis,N.M.,RRothstein,J.H.,IPejaver,V.,DMiddha,S.,IMcDonnell,S.K.,IBaheti,S.,IMusolf,A.,,

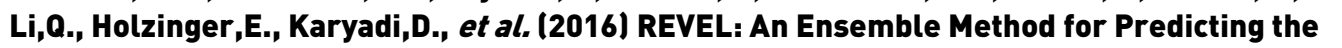
Pathogenicity[fRare[Missense[Variants.TheLAmerican[DournalWfLHumanWenetics,D9,B77885.

14.Lake,N.J.,WWebb,B.D.,[Stroud,D.A.,,Richman,T.R.,,Ruzzenente,B.,[Compton,A.G.,D

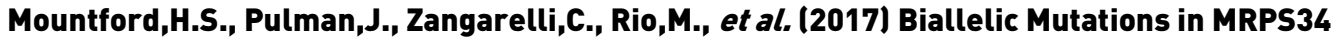

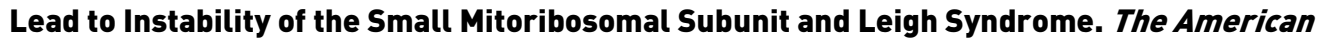
Journal[FfHumanEenetics, [1 01,[239-254.] 


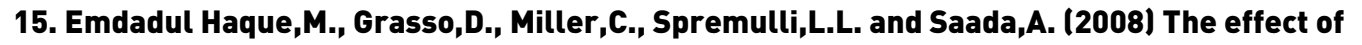

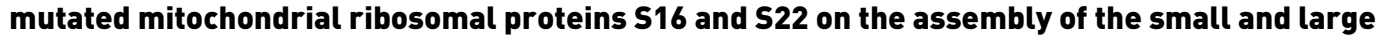
ribosomal[Eubunits[nthuman[mitochondria.DMitochondrion,[B,[254-261.]

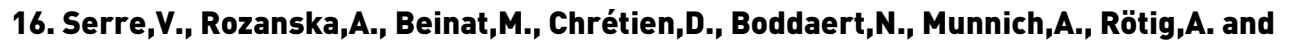
Chrzanowska-Lightowlers,Z.M.[2013)[Mutations[n[mitochondrial[ribosomal(protein[MRPL12D leads[1o[growth[retardation, [heurological[ieterioration[and[mitochondrial[iranslation deficiency.BBiochim.BBiophys.[Acta,[1832,[1304-1312.]

17.Miller,C.,[Saada,A.,[Shaul,N.,[Shabtai,N.,Ben-Shalom,E.,[Shaag,A.,[Hershkovitz,E.End

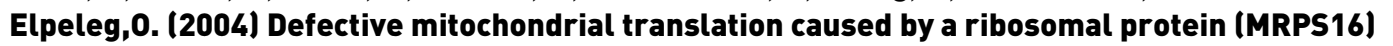
mutation.[Ann.LNeurol,,[56, [734-738.0

18.BBaertling,F.,[Haack,T.B.,RRodenburg,R.J.,[Schaper,J.,[Seibt,A.,[Strom,T.M.,LMeitinger,T.,D

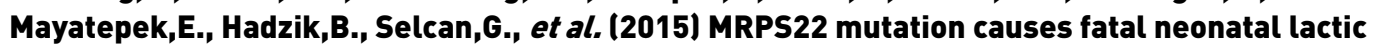

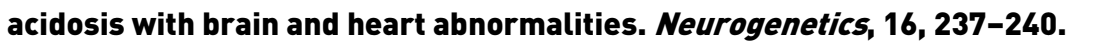

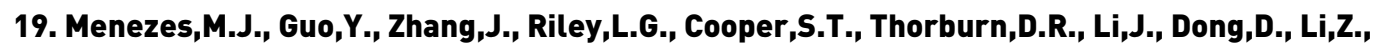

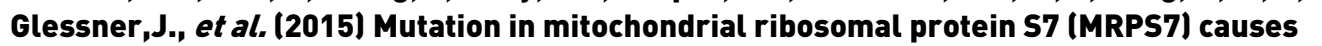

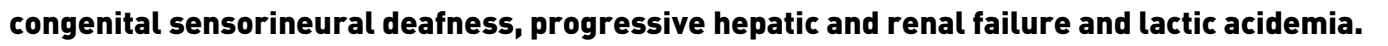
Hum.LMol.LGenet.,[24,[2297-2307.]

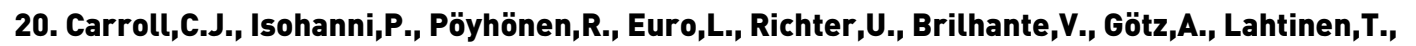

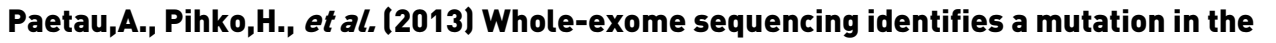
mitochondrial[ribosomeゆroteinTMRPL4410[underlie[mitochondrial[nfantile[rardiomyopathy. J.LMed.LEenet., ,E0,151-159.0

21.Bogenhagen,D.F.,(Dstermeyer-Fay,A.G.,[Haley,J.D.End[Garcia-Diaz,M.L2018)[Kinetics(and]

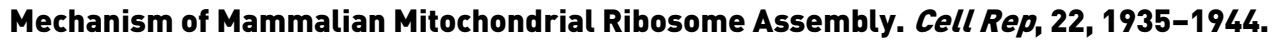

22.[Kummer,E.,Leibundgut,M.,Rackham,0.,Lee,R.G.,Boehringer,D.,IFilipovska,A.EndIBan,N.]

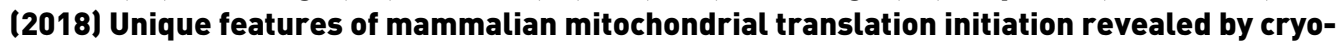
EM.DNature,[10.1038/s41586-018-0373-y.

23.TKaushal,P.S.,[Sharma,M.R.,BBooth,T.M.,[Haque,E.M.,Tung,C.-S.,[Wanbonmatsu,K.Y.,, Spremulli,L.L.End[Agrawal,R.K.L2014)[ryo-EM[structure[bfithe[small[subunit[ffihe[ mammalian[mitochondrial[ribosome.DProc.LNatl.LAcad.LSCi.W.S.A.,[11, W7284-7289.]

24.[Gardeitchik,T.,IMohamed,M.,Ruzzenente,B.,IKarall,D.,EGuerrero-Castillo,S.,Dalloyaux,D.,Eran口

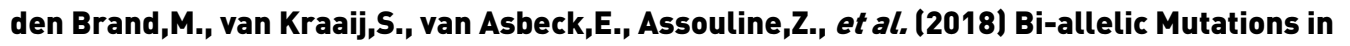
the[MitochondrialRibosomal[ProteinIMRPS2 [ause[Sensorineural[HearingLoss, $\square$

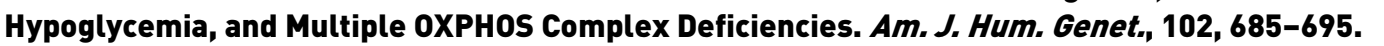

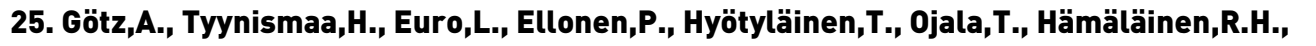

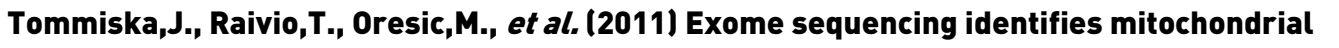
alanyl-tRNA[3ynthetase[mutations[n[nfantile[mitochondrial[ardiomyopathy.[Am. D. [Hum.D Genet.,B8, $635-642.0$

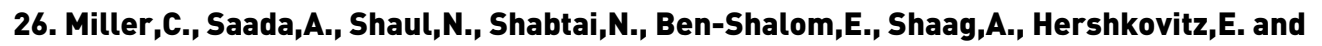

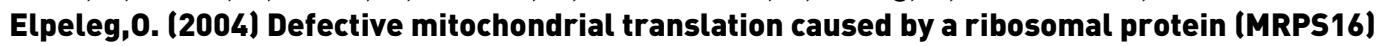
mutation.DAnn.LNeurol.,W6, 1734-738.0

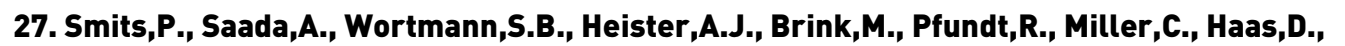
Hantschmann,R.,Rodenburg,R.J.T.,[et[al.L2011)[Mutation[n[mitochondrial[ribosomal[proteinD

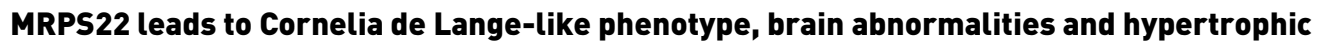

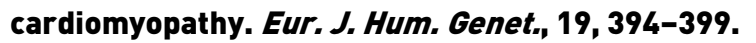




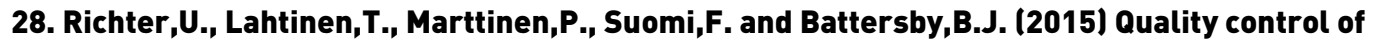

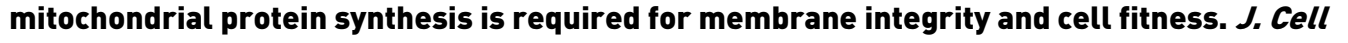
Biol.,[211,[B73-389.]

29.Berger,T.,[Brigl,M.,[Herrmann,J.M.,LVielhauer,V.,Luckow,B.,[Schlondorff,D.WndIKretzler,M.D

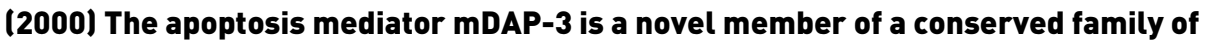

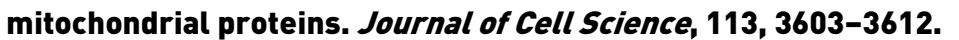

30.Rightowlers,R.N.,[Rozanska,A.Tnd[Chrzanowska-Lightowlers,Z.M.12014)[Mitochondrial protein[synthesis:[Figuring [The[iundamentals, [romplexities[and[Komplications, [ff[mammalian mitochondrial[translation.[FEBSLetters, [588,[2496-2503.]

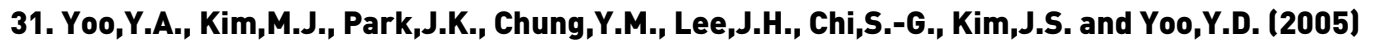

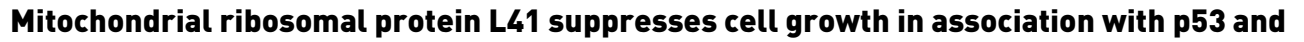
p27Kip1.DMolecularlandCellularBiology,[25,16603-6616.0

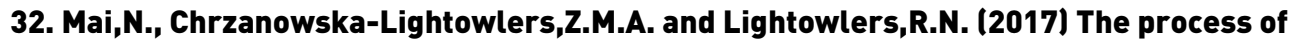
mammalian[mitochondrial|protein[synthesis.[Cell[TissueLRes.,[B67,[E-20.0

33.Richter,U.,Lahtinen,T.,Larttinen,P.,LMyöhänen,M.,[Greco,D.,Cannino,G.,Dacobs,H.T.,D

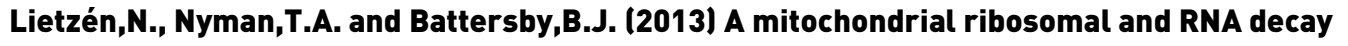

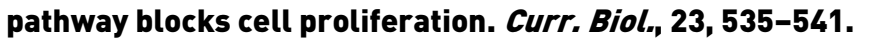

34.Dackson,C.B.,WNuoffer,J.M.,[Hahn,D.,Prokisch,H.,LHaberberger,B.,[Gautschi,M.,LHaberli,A.,D

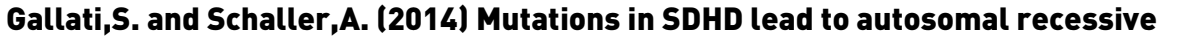

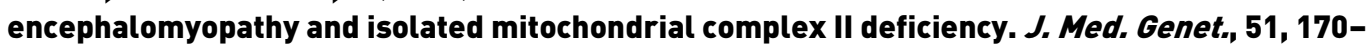
175.

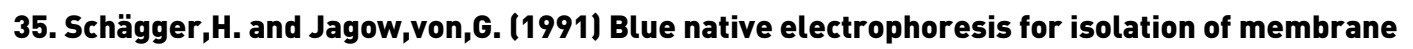
protein[tomplexes[n[enzymatically[ictive[form.[Anal.BBiochem.,[199,[223-231.]

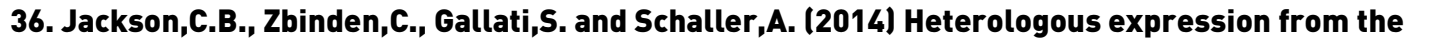
humanD-Loop[nநrganello.DMitochondrion, [7, 167-75.]

37.Dackson,C.B.,Weuwirth,C.,[Hahn,D.,WNuoffer,J.M.,[Frank,S.,[Gallati,S.TindWchaller,A.12014)[

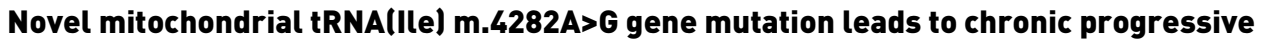

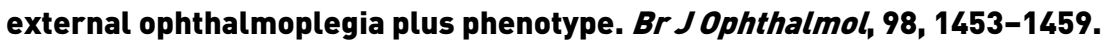

38.[Galmiche,L.,[Werre,V.,BBeinat,M.,[Assouline,Z.,Lebre,A.-S.,[Chrétien,D.,Wietschke,P.,Benes,V.,,

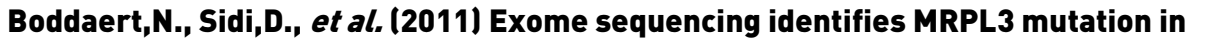
mitochondrial[Fardiomyopathy.[Hum.LMutat,,[B2,[1225-1231.]

39.Distelmaier,F.,[Haack,T.B.,[Catarino,C.B.,[Gallenmüller,C.,LRodenburg,R.J.,[Strom,T.M.,,

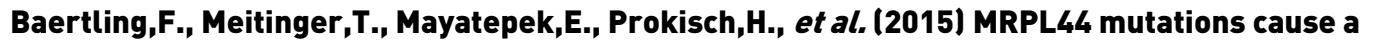
slowlyధrogressive[multisystem[isease[with[thildhood-onset Thypertrophictrardiomyopathy. $\square$ Neurogenetics, [6, B19-323.0

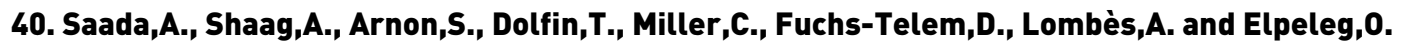
(2007)[Antenatal[mitochondrial[disease[raused [by[nitochondrial[ribosomal[proteinपMRPS22] $\square$ mutation.‥LMed.LGenet.,Z.4,, $1784-786.0$

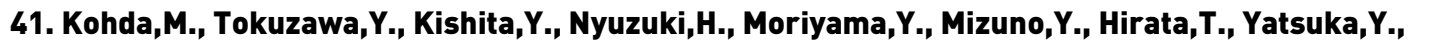

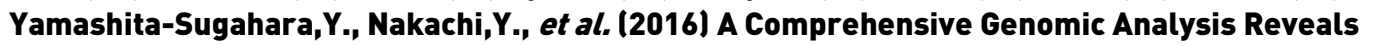

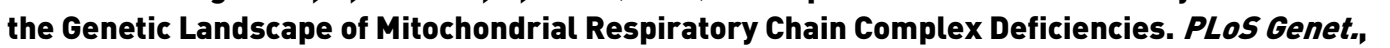
12,[E1005679-31. 


\section{Legends to figures}

Figure 1. Index patient and targeted sequencing reveals mutations in MRPS14. (A) Index patient shows discrete facial features of hypertelorism, flat nasal bridge, a small midface region with retroverted and low set ears. (B) Pedigree. Index patient (arrow) was born to consanguineous parents. Unaffected heterozygous carriers of the mutant allele are indicated by the half black symbols and the affected individual is indicated by the solid black symbol. (C) Electropherograms show the confirmation of the homozygous c.322C $>\mathrm{T}$ in exon 3 of MRPS14 by Sanger sequencing predicting a p.(Arg108Cys) change. Sanger sequencing was performed in the index patient and its parents, who were both heterozygous carriers. Both parents are clinically unaffected carriers of the same heterozygous variant c.322C $>\mathrm{T}$ in MRPS14, whereas the clinically affected index patient is homozygous for the c.322C $>\mathrm{T}$ variant. (D) MRPS14 mutation analysis shows conservation among different species. Indicated with the arrow is the amino acid change arginine to cysteine at position 108 resulting from the $\mathrm{c} .322 \mathrm{C}>\mathrm{T}$ transition.

A

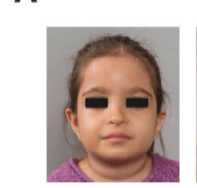

B

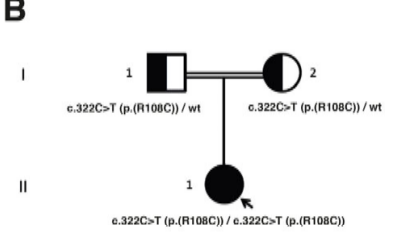

D

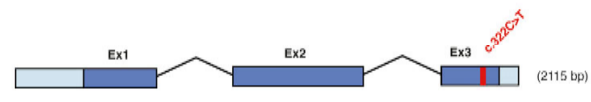
MRPS14

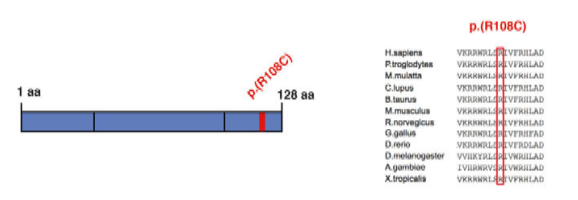

C
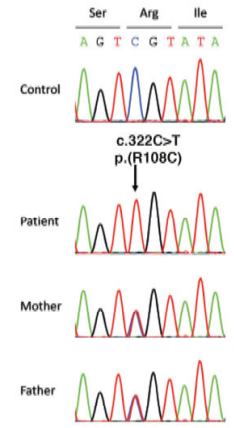
Figure 2. MRPS14 mutated fibroblasts present with multiple respiratory complex

deficiency. (A) Immunoblot of blue-native respiratory chain complex analysis from total cell lysates of control and patient cells. Patient cells show drastically reduced assembled complex I, IV and V, reduced complex III with unchanged complex II. (B) Immunoblots of steadystate mitochondrial respiratory chain complex subunits reveals multiple OXPHOS deficiency in patient fibroblasts with signficant reduction of $\mathrm{CI}$, CIV and CV. Graph represents immunoblot quantification of average of groups with standard deviation. Vinculin was used as loading control for normalisation. (C) Immunoblots of steady-state mitochondrial ribosomal proteins of the small (SSU: MRPS10, MRPS18B, MRPS27) and large (LSU: MRPL11, MRPL37, MRPL44) subunits remain unchanged apart from a two-fold increase in uS14m in patient cells. Graph represents immunoblot quantification of average of groups with standard deviation. Vinculin was used as loading control for normalisation.

Controls represent fibroblast lines derived from healthy individuals in two biological replicates (subculturings); patient represents four biological replicates (subculturings). Unpaired t-test, statistical significance: $\mathrm{n}$. s. (not significant), ${ }^{*} \mathrm{p} \leq 0.05,{ }^{* *} \mathrm{p} \leq 0.01$

A

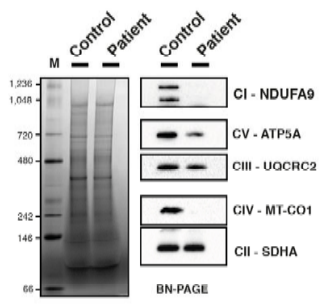

C

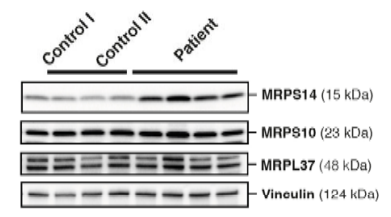

B
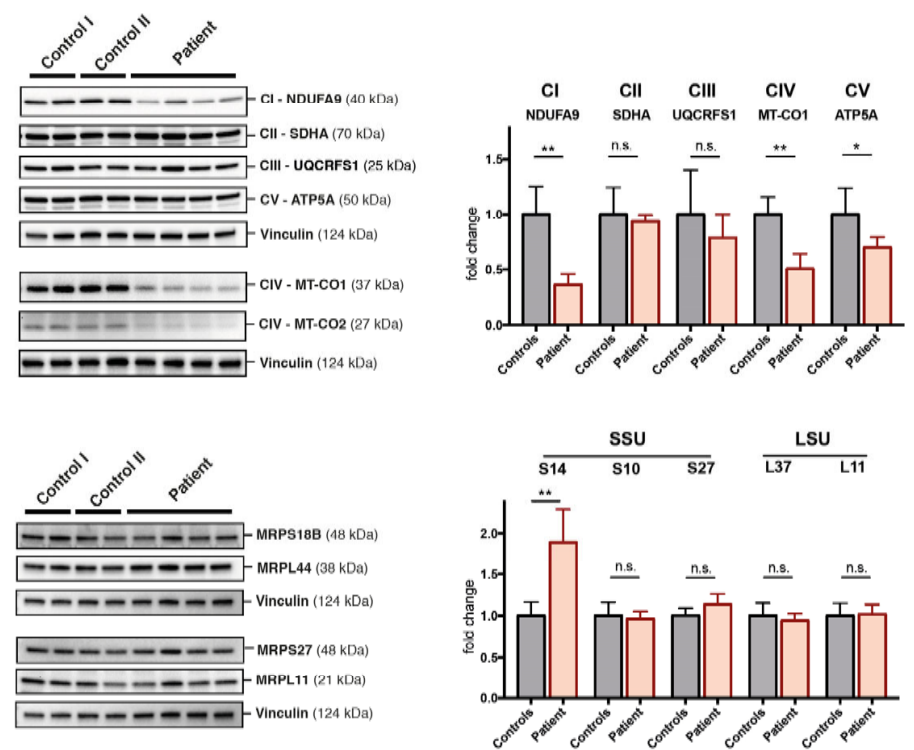
Figure 3. Mitochondrial translation capacity and ribosome assembly state in patient

fibroblasts. (A) Immunoblotting of fractions from linear 10\%-30\% sucrose density gradients of control and patient fibroblasts. Probing against SSU subunits uS14m, MRPS27 and MRPS18B reveal an equal distribution of 28S to fully assembled 55S between control and patient with no particular assembly intermediates or gross assembly defect in the patient. A fraction of input for the sucrose gradient was used as control (IN). Numbers indicate respective fractions collected from top to bottom. (B) Northern blot analysis of total RNA from control $(n=3)$ and patient cells from three subculturings for $12 \mathrm{~S}$ and $16 \mathrm{~S}$ rRNAs and MT-ATP6/8 transcript reveal no signficant changes in the patient (quantification Fig.S3A). (C) Quantitative PCR of mitochondrial transcripts (12S rRNA, 16S rRNA, mt-ND5, mtCOX1) in controls and patient cells reveal no significant changes in 12S rRNA and replicate Northern blot findings. Controls represent three fibroblast lines derived from healthy individuals; patient represents four biological replicates (subculturings). Graph represents group average (with duplicate runs for $12 \mathrm{~S}$ and $16 \mathrm{~S}$ rRNA). $\beta$-actin was used for normalisation. (D) ${ }^{35} \mathrm{~S}$-metabolic labelling reveals a general decrease in mitochondrial translation in patient fibroblasts (30 min pulse). Coomassie staining was performed as loading control. (E) Quantification of ${ }^{35} \mathrm{~S}$-met/cys incorporation in patient relative to control from three independent experiments performed in biological duplicates. Graph represents quantification of ${ }^{35} \mathrm{~S}$ incorporation of average of groups with standard deviation. Coomassie was used as loading control for normalisation. Unpaired t-test, statistical significance: n.s. (not significant), ${ }^{*} \mathrm{p} \leq 0.05,{ }^{* *} \mathrm{p} \leq 0.01, * * * \mathrm{p} \leq 0.001$ 
A
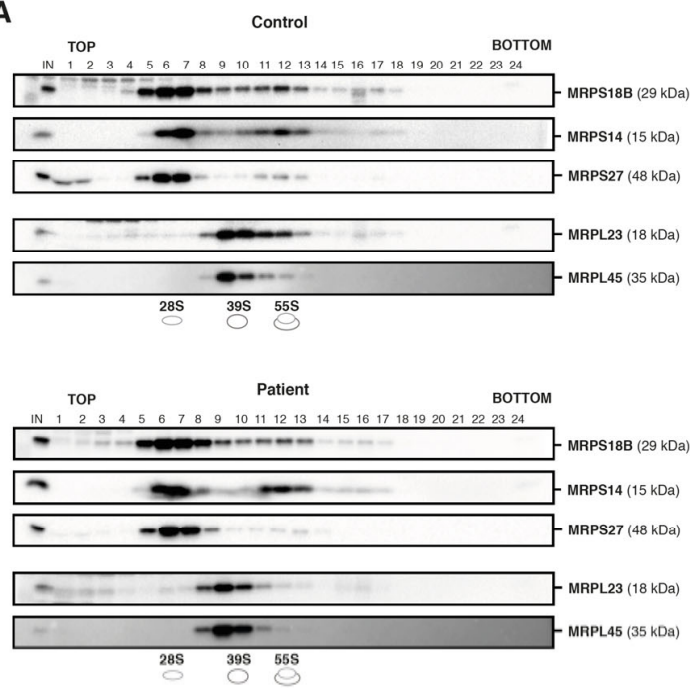

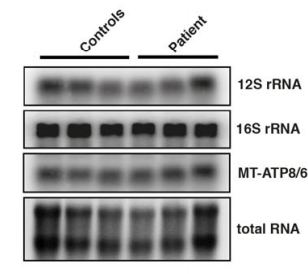

C

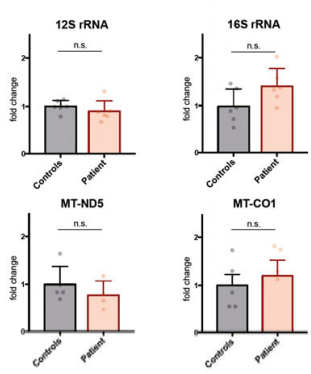

D

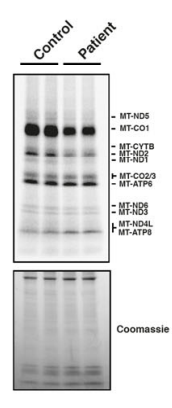

E

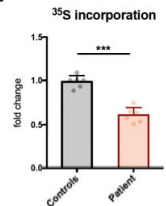


Figure 4. Modelling of uS14m mutation R108C within the 55S mitochondrial initiating

ribosome. (A) Human mitochondrial initiating ribosome structure (pdb 6GAW) (4): the ribosomal proteins from the small subunit are shown in yellow and the 12S rRNA is shown in orange. The ribosomal proteins from the large subunit are shown in blue and the 16S rRNA is shown in green. The mRNA is shown in black and the tRNA in magenta. The mitochondrial initiation factor mtIF2 is shown in dark green. Ribosomal protein $\mathrm{uS} 14 \mathrm{~m}$ is shown in red. On the left, the complete structure of the initating 55S ribosome is shown, on the right, the large subunit has been removed. The black frame indicates the part of the structure that is magnified and shown in (B). This magnification shows the position of the small ribosomal subunit protein $\mathrm{uS} 14 \mathrm{~m}$ in red in respect to the tRNA anticodon (in magenta) and the mRNA (in black) in the mRNA channel. The lateral chain of Arg108 is also shown in red. (C) Arg108 lateral chain interacts with ribosomal protein $\mathrm{uS} 10 \mathrm{~m}$ and the sugar phosphate backbone from A579 of 12S rRNA. (D) By modelling, the lateral chain of residue 108 has been replaced by a cysteine lateral chain thereby illustrating the probable structural consequences of the $\mathrm{R} 108 \mathrm{C}$ mutations and the loss of the previously mentioned interactions with ribosomal protein uS10m and 12S rRNA (A579). 
A
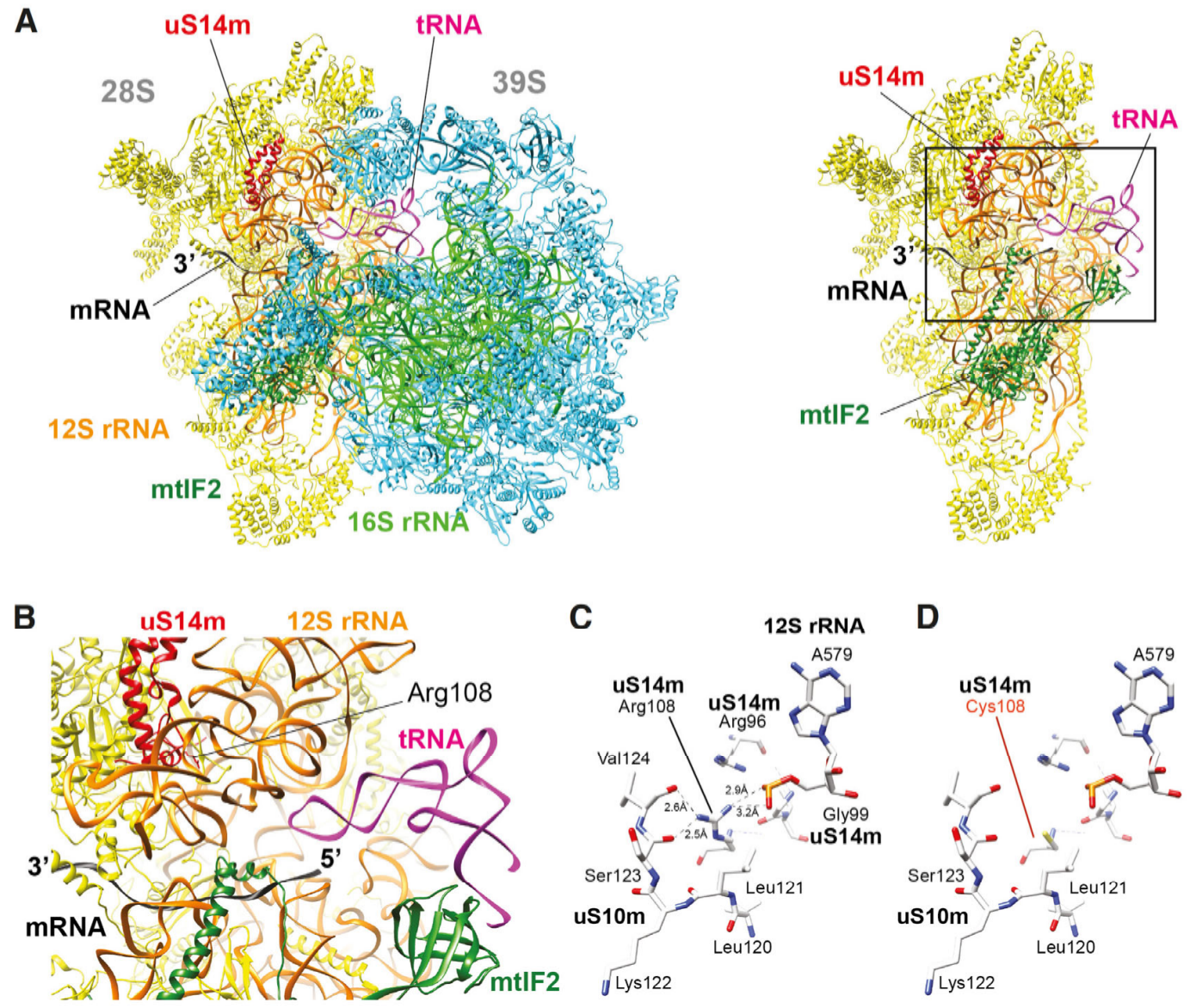
Figure 5. Functional rescue of MRPS14-deficient fibroblasts corrects mitochondrial

translation phenotype. (A) Immunoblots of steady-state levels of respiratory chain and mitochondrial ribosomal subunit from control and patient fibroblasts transduced with wildtype (WT) or mutant (+R108C) MRPS14. Patients show reduced steady-state level of MTCO1 compared to controls (non-transduced cells). Stably transduced clones expressing mutant MRPS14 (+R108C) show decrease steady-state levels of MT-CO1 in both control and patient with additive effect in patient cells. Transduction with wild-type MRPS14 rescues MT-CO1 and MT-CO2 levels in patient fibroblasts confirming the pathogenicity of the R108C mutation. Expression levels of MRPS14 in stably transduced cells reveal partial overexpression with additive effect in patient cells. Mitochondrial complex II subunit SDHA, mitochondrial ribosomal small (MRPS27) and large (MRPL44) subunits remain seemingly unaffected. Samples are represented in technical duplicates and Vinculin and stain-free total protein serve as loading control. (B) ${ }^{35} \mathrm{~S}$-metabolic labelling of patient cells stably transduced with wild-type cDNA of MRPS14 show increased translation rates in patient cells and corroborates rescued steady-state MT-CO1/2 levels in (A). Samples are represented in biological duplicates and coomassie serves as laoding control.

\section{$\mathrm{p}$}

A

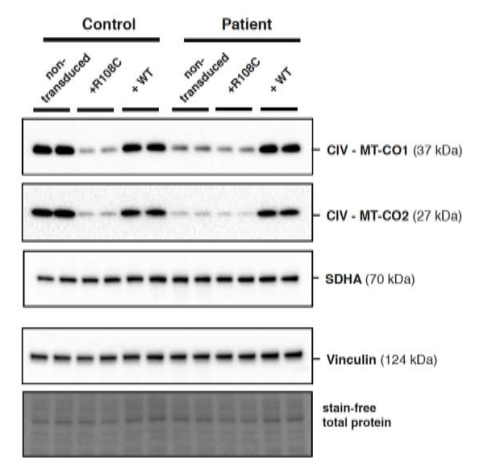

B

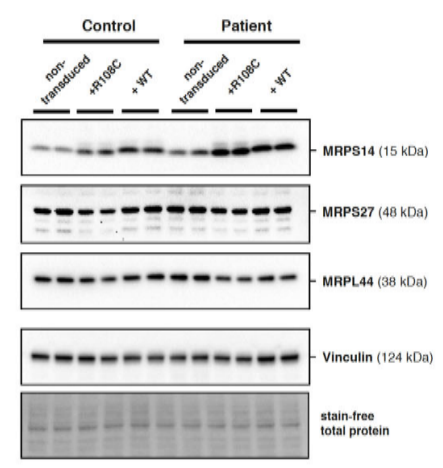


Tables

Table I Disease-associated mitochondrial ribosomal genes

\begin{tabular}{|c|c|c|c|}
\hline MRPL & Phenotype & Mutation & Reference \\
\hline $\begin{array}{l}\text { MRPL3 } \\
\text { MRPL12 } \\
\text { MRPL44 }\end{array}$ & $\begin{array}{l}\text { HCM, psychomotor retardation } \\
\text { growth retardation, neurological deterioration } \\
\text { infantile cardiomyopathy }\end{array}$ & $\begin{array}{l}\text { c. } 950 \mathrm{C}>\mathrm{G}(\text { p.Pro317Arg }) / \text { large deletion } \\
\text { c. } 542 \mathrm{C}>\mathrm{T} \text { (p.Ala181Val) } \\
\text { c. } 467 \mathrm{C}>\mathrm{G} \text { (p.Leu156Arg) }\end{array}$ & $\begin{array}{l}(38) \\
(16) \\
(20,39)\end{array}$ \\
\hline MRPS & Phenotype & Mutation & Reference \\
\hline MRPS2 & hypotonia, hyperlacticacidemia, growth retardation & $\begin{array}{l}\text { c. } 328 \mathrm{C}>\mathrm{T} \text { (p.Arg110Cys) } \\
\text { c. } 340 \mathrm{G}>\mathrm{A} \text { (p.Asp114Asn) } \\
\text { c. } 413 \mathrm{G}>\mathrm{A} \text { (p.Arg138His) }\end{array}$ & (24) \\
\hline MRPS7 & hepatic and renal failure, sensorineural deafness & c. $550 \mathrm{~A}>\mathrm{G}$ (p.Met184Val) & (19) \\
\hline MRPS14 uS14m & HCM, cachexia, lactic acidosis & c.322C $>$ T (p.Arg108Cys) & this report \\
\hline MRPSI6 & $\begin{array}{l}\text { fatal lactic acidosis, } \mathrm{CM} \\
\text { tubulopathy, hypotonia, skin edema }\end{array}$ & c. $331 \mathrm{C}>\mathrm{T}\left(\mathrm{p} . \operatorname{Arg} 111^{*}\right)$ & $(15,26)$ \\
\hline MRPS 22 & $\begin{array}{l}\mathrm{CM} \text {, tubulopathy, hypotonia, skin edema } \\
\text { Cornelia de Lange-like dysmorphic features } \\
\text { encephalocardiomyopathy } \\
\text { hepatic disease }\end{array}$ & $\begin{array}{l}\text { c. } 509 \mathrm{G}>\mathrm{A}(\mathrm{p} . \text { Arg } 170 \mathrm{Cys}) \\
\text { c. } 644 \mathrm{~T}>\mathrm{C} \text { (p.Leu215Pro) } \\
\text { c. } 119 \mathrm{C}>\mathrm{G} \text { (p.Pro40Arg) }\end{array}$ & $(15,18,27,40)$ \\
\hline MRPS34 & $\begin{array}{l}\text { Leigh syndrome, hyperlacticacidemia } \\
\text { microcephaly, transient metabolic acidosis }\end{array}$ & $\begin{array}{l}\text { c.321+1G }>\text { T (p.Val100_Gln107del) } \\
\text { c.322-10G }>\text { A, ((p.Asn108Leufs*12) } \\
\text { c.37G }>\text { A (p.Glu13Lys), c.94C }>\text { T (p.Gln32*) }\end{array}$ & (14) \\
\hline
\end{tabular}

Abbreviations: MRPS - mitochondrial ribosomal protein small subunit, MRPL - mitochondrial ribosomal protein large subunit

HCM hypertrophic cardiomyopathy, CM cardiomyopathy 